\title{
Overexpression of S100A4 is closely associated with the progression and prognosis of gastric cancer in young patients
}

\author{
HUA LI $^{1}$, ZIQUAN LIU ${ }^{2,3}$, CHUANXIANG XU $^{2}$, YUNYUN CHEN ${ }^{2}$, JIANWEI ZHANG ${ }^{2}$, BO CUI $^{2}$, \\ XUEWEI CHEN ${ }^{2}$, GAIHONG AN ${ }^{2}$, XIAOJUN SHE ${ }^{2}$, HONGTAO LIU ${ }^{2}$, ZIFENG JIANG $^{4}$ and TIANHUI WANG ${ }^{2}$ \\ ${ }^{1}$ Endoscopy Division, Tianjin Medical University Cancer Hospital and City Key Laboratory of Tianjin Cancer Center, \\ Tianjin 300060; ${ }^{2}$ Institute of Health and Environmental Medicine, Tianjin 300050; ${ }^{3}$ Department of Physiology and \\ Pathophysiology, Logistics College of Chinese People's Armed Police Force, Tianjin 300162; ${ }^{4}$ Institute of Zoology, \\ Chinese Academy of Sciences, Chaoyang, Beijing 100101, P.R. China
}

Received November 15, 2012; Accepted February 13, 2013

DOI: $10.3892 / 01.2013 .1220$

\begin{abstract}
The aim of this study was to determine the correlation of S100A4 expression with the progression, prognosis and clinical pathology of gastric cancer (GC) in young pateints. A total of 85 tumor tissues with corresponding adjacent normal tissues and 62 non-metastatic lymph nodes (LNs) with corresponding metastatic LNs were obtained from young GC patients ( $<40$ years old) who underwent surgery between January 2001 and December 2006. The expression of S100A4 was detected by RT-PCR and immunohistochemistry. Differences in the expression of S100A4 mRNA or protein were observed among the GC tissues, matched normal gastric mucosa, non-metastatic LNs and metastatic LNs. The expression of S100A4 mRNA and protein in GC tissues and metastatic LNs was significantly higher compared with that in the matched normal gastric mucosa and non-metastatic LNs, respectively $(\mathrm{P}<0.05)$. The overexpression of S100A4 was significantly associated with parameters involved in tumor progression and poor prognosis, including tumor size $(\mathrm{P}=0.017)$, Lauren classification $(\mathrm{P}=0.002)$, histological classification $(\mathrm{P}=0.010)$, histological differentiation $(\mathrm{P}=0.000)$, Borrmann classification $(\mathrm{P}=0.020)$, tumor-node-metastasis (TNM) stage $(\mathrm{P}=0.000)$, LN metastasis $(\mathrm{P}=0.000)$ and distant metastasis $(\mathrm{P}=0.024)$. Multivariate analysis suggested that patient age $(\mathrm{P}=0.035)$, tumor size $(\mathrm{P}=0.002)$, TNM stage $(\mathrm{P}=0.001)$ and $\mathrm{S} 100 \mathrm{~A} 4$ upregulation $(\mathrm{P}=0.000)$ were independent prognostic indicators for the disease. The overexpression of S100A4 in young GC patients is significantly associated with the clinicopathological characteristics. S100A4 may
\end{abstract}

Correspondence to: Professor Tianhui Wang, Institute of Health and Environmental Medicine, 1 Dali Road, Tianjin 300050, P.R. China

E-mail: wydny668@163.com

Key words: S100A4, gastric cancer, young patients, progression, prognosis, immunohistochemistry be used as a biomarker to predict the progression and poor prognosis of GC in young patients.

\section{Introduction}

Gastric cancer (GC) is an extremely common disease worldwide and the second most common cause of cancer-related mortality (1). GC is considered to be a disease of the middle aged and elderly. However, a previous study revealed that 2 to $15 \%$ of patients with GC are younger than 40 years of age (defined as young GC patients), and that the relative proportion of young GC patients is higher than that of older GC patients (2). Despite the advances in chemotherapy and surgical techniques, the overall 5-year survival rate of GC patients in China remains low (3). At present, clinical staging and histopathological criteria are the only parameters used to stratify patients. However, the current staging classifications do not produce accurate predictions of patient outcomes. Molecular biomarkers may account for this diversity and several prognostic factors have been identified (4). However, none of these methods have been proven to be robust enough to be incorporated into routine practice.

S100A4 belongs to the S100 family, which is involved in the regulation of a wide range of intracellular and extracellular biological functions, including cell motility, differentiation and contractility, and is classified as a metastasis-related gene (5). A number of studies have suggested that S100A4 overexpression is correlated with poor clinical outcomes in various human cancers, such as bladder (6), colorectal (7), ovarian (8) and esophageal carcinoma (9). In the present study, immunohistochemistry (IHC) and RT-PCR were used to analyze the expression of S100A4 in 85 clinicopathologically characterized young GC patients.

\section{Patients and methods}

Patient population. A total of 85 young GC patients treated at Tianjin Medical University Cancer Hospital and Institute of China (Tianjin, China) between January 2001 and December 2006 were included in the study at the time 
of primary surgery for GC. The study was approved by the Ethics Committee of the hospital and informed consent was obtained from the patients. Patients with any of the following conditions were excluded from the present study: older than 40 years old, histology other than adenocarcinoma, preoperative chemoradiotherapy, mortality not caused by cancer and unknown stage of disease. The study population included 85 patients in tumor-node-metastasis (TNM) stages I-IV who had undergone surgery. Paraffin sections of tissues from these patients were prepared. The presence or absence of distant metastases was determined through radiological examination. Primary tumor sections were re-evaluated by an experienced pathologist who was blinded to the patients' survival or other clinical variables to ensure consistent staging and grading. Representative sections for each tumor were identified and prepared for subsequent IHC analysis.

IHC and scoring. IHC staining was performed based on a previously described method (10) to identify changes in the protein expression of the primary GC, metastatic $\mathrm{LN}$ and their normal counterpart tissues. In brief, the slides were placed in an oven at $60^{\circ} \mathrm{C}$ for $2 \mathrm{~h}$, deparaffinized with xylene and then rehydrated. The sections were submerged in an EDTA antigenic retrieval buffer, placed in a microwave for antigen retrieval, treated with 3\% hydrogen peroxide in methanol to quench endogenous peroxidase activity and then incubated with $1 \%$ bovine serum albumin to block non-specific binding. The sections were incubated with mouse anti-S100A4 (Santa Cruz Biotechnology, Santa Cruz, CA, USA) overnight at $4^{\circ} \mathrm{C}$. Normal goat serum was used as the negative control. The tissue sections were washed, treated with secondary antibody, counterstained with hematoxylin, dehydrated and then mounted.

S100A4 was stained yellow-brown in the cytoplasm and nucleus. The degree of immunostaining was reviewed and scored independently by two observers based on the staining intensity and percentage of positive cells. The intensity grading scale was according to the following criteria: 0 (no staining), 1 (weak staining, light yellow), 2 (moderate staining yellow-brown) and 3 (strong staining, brown). Moderate and strong staining indicated tumors with high S100A4 expression, while no and weak staining indicated low S100A4 expression.

$R T$-PCR. Total RNA was extracted from the primary GC, metastatic LN and their normal counterpart tissues using TRIzol reagent (Takara Biotech, Dalian Co., Ltd., Otsu, Japan). Reverse transcriptase reactions were performed using $1 \mathrm{mg}$ of total RNA and PrimeScript ${ }^{\mathrm{TM}}$ RT reagent kit (Takara Biotech) followed by PCR amplification (TC-412 thermal cycler; Techne, Stone, Staffordshire, UK) with specific primers. PCR amplification was performed as follows: 30 cycles of $94^{\circ} \mathrm{C}$ for $5 \mathrm{~min}, 58^{\circ} \mathrm{C}$ for $30 \mathrm{sec}$ and $72^{\circ} \mathrm{C}$ for $30 \mathrm{sec}$, with a final extension of $72^{\circ} \mathrm{C}$ for $10 \mathrm{~min}$. After the PCR products were electrophoresed on $1.5 \%$ agarose gels, they were arrayed using a Bio-Rad scanner system (Hercules, CA, USA) and analyzed with Quantity One software. The specific primers for the S100A4 gene were S100A4-sense (5'-GATGTGATGGTGTCCAccTT-3') and S100A4-antisense (5'-ATTTCTTCCTGGGCTGCTTA-3') whose target was a 277 -bp fragment. Primer pairs specific to the $\beta$-actin gene ( $\beta$-actin-sense, 5'-CCAGATCAtGTTTGAGACCT-3'; $\beta$-actin-antisense, 5'-TTGAAGGTAGTTTCGTGGAT-3'; PCR product, $480 \mathrm{bp}$ ) served as the internal standard.

Postoperative follow-up. Following surgery, each patient was scheduled for a follow-up examination every 4 months in the first year, semi-annually in the second year and annually thereafter. More frequent examinations were scheduled if clinically indicated. The cause of mortality was registered and classified as mortality due to GC, other causes or unknown causes. For overall survival, the median follow-up of the surviving patients was 15 months (range, 2-81 months).

Statistical analysis. The associations between S100A4 staining and clinicopathological variables were tested using the two-tailed Fisher's exact test or linear-by-linear association Chi-squared test. The Kaplan-Meier method was used to calculate the survival functions and differences were assessed with the log-rank test. Multivariate analysis was performed using the Cox proportional hazards regression model. Overall and disease-specific survival were determined from the time of surgery until mortality. $\mathrm{P}<0.05$ was considered to indicate statistically significant differences. All reported P-values were two-sided and all analyses were performed using the Statistical Package for Social Sciences, version 19.0, for Windows (SPSS Inc., Chicago, IL, USA).

\section{Results}

\section{Expression of S100A4}

Expression of S100A4 in the gastric mucosa. S100A4 protein was detected in 18 (21.18\%) out of the 85 non-tumor mucosal samples obtained from young patients. The majority of the samples expressed the protein at a low level. The expression was negligible or negative in the normal gastric mucosa but moderate to strong in lymphocytes and smooth muscles cells. Among the $85 \mathrm{GC}$ tissue specimens from young patients screened for S100A4 protein expression, 53 (62.35\%) exhibited S100A4 overexpression, in which immunostaining was observed in the cytoplasm or the nucleus of the tumor cells (Figs. 1-4). Statistically, S100A4 overexpression was closely associated with the tumor size $(\mathrm{P}=0.017)$, Lauren classification $(\mathrm{P}=0.002)$, histological classification $(\mathrm{P}=0.010)$, histological differentiation $(\mathrm{P}=0.000)$, Borrmann classification $(\mathrm{P}=0.020)$, TNM stage $(\mathrm{P}=0.000)$, $\mathrm{LN}$ metastasis $(\mathrm{P}=0.000)$ and distant metastasis $(\mathrm{P}=0.024)$. However, no significant correlation was observed between S100A4 overexpression and other clinicopathological parameters, including age, gender, tumor location, surgery type and Heliobacter pylori infection (Table I).

Expression of S100A4 in the LN. The S100A4 protein was present in non-metastatic LNs at low levels (Fig. 2). The S100A4 protein level in the metastatic LNs was significantly higher than that of the non-metastatic LNs. S100A4 overexpression was closely correlated with $\mathrm{LN}$ staging $(\mathrm{P}=0.000$; Table II). No significant difference in S100A4 expression was observed between the GC tissues and metastatic LNs $(\mathrm{P}=0.896)$.

Expression of S100A4 mRNA. S100A4 expression was detected using IHC. The relative expression level of $\beta$-actin/S100A4 in the primary GC $(0.4493 \pm 0.0453)$ was higher than that in the normal tissues $(0.1145 \pm 0.1000)$. In addition, the relative 
Table I. Association between S100A4 expression and clinicopathological characteristics.

\begin{tabular}{|c|c|c|c|c|}
\hline \multirow[b]{2}{*}{ Characteristics } & \multicolumn{2}{|c|}{ S100A4 } & \multirow[b]{2}{*}{$\chi^{2}$} & \multirow[b]{2}{*}{ P-value } \\
\hline & Low & High & & \\
\hline Age (years) & & & 0.134 & 0.935 \\
\hline$<30$ & 6 & 11 & & \\
\hline $31-35$ & 9 & 16 & & \\
\hline $36-40$ & 17 & 26 & & \\
\hline Gender & & & 1.011 & 0.315 \\
\hline Male & 15 & 19 & & \\
\hline Female & 17 & 34 & & \\
\hline Tumor location & & & 0.894 & 0.640 \\
\hline Proximal & 5 & 11 & & \\
\hline Middle & 10 & 12 & & \\
\hline Distal & 17 & 30 & & \\
\hline Tumor size $(\mathrm{cm})$ & & & 5.647 & 0.017 \\
\hline$<5$ & 18 & 16 & & \\
\hline$\geq 5$ & 14 & 37 & & \\
\hline Surgery & & & 3.348 & 0.341 \\
\hline Distal subtotal gastrectomy & 17 & 28 & & \\
\hline Proximal subtotal gastrectomy & 5 & 9 & & \\
\hline Total gastrectomy & 9 & 9 & & \\
\hline Other & 1 & 7 & & \\
\hline Lauren classification & & & 9.465 & 0.002 \\
\hline Intestinal & 17 & 11 & & \\
\hline Diffuse & 15 & 42 & & \\
\hline Histological classification & & & 13.326 & 0.010 \\
\hline Tubular adenocarcinoma & 14 & 10 & & \\
\hline Papillary adenocarcinoma & 5 & 3 & & \\
\hline Mucinous adenocarcinoma & 7 & 11 & & \\
\hline Poorly differentiated adenocarcinoma & 6 & 25 & & \\
\hline Other & 0 & 4 & & \\
\hline Histological differentiation & & & 18.501 & 0.000 \\
\hline Well & 8 & 1 & & \\
\hline Moderately & 7 & 3 & & \\
\hline Poorly & 17 & 49 & & \\
\hline Borrmann classification (type) & & & 9.890 & 0.020 \\
\hline I & 6 & 1 & & \\
\hline II & 11 & 13 & & \\
\hline III & 11 & 27 & & \\
\hline IV & 4 & 12 & & \\
\hline Tumor-node-metastasis stage & & & 19.178 & 0.000 \\
\hline I & 9 & 1 & & \\
\hline II & 4 & 1 & & \\
\hline III & 8 & 17 & & \\
\hline IV & 11 & 34 & & \\
\hline Lymph node metastasis & & & 27.400 & 0.000 \\
\hline No & 12 & 2 & & \\
\hline N1 & 7 & 4 & & \\
\hline $\mathrm{N} 2$ & 10 & 19 & & \\
\hline N3 & 3 & 28 & & \\
\hline Distant metastasis & & & 5.070 & 0.024 \\
\hline M0 & 31 & 46 & & \\
\hline M1 & 0 & 8 & & \\
\hline H.pylori & & & 3.108 & 0.078 \\
\hline$(+)$ & 13 & 12 & & \\
\hline$(-)$ & 19 & 41 & & \\
\hline
\end{tabular}


A
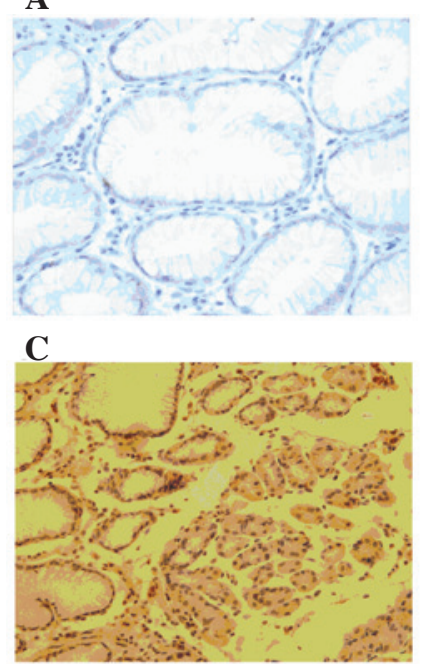

B

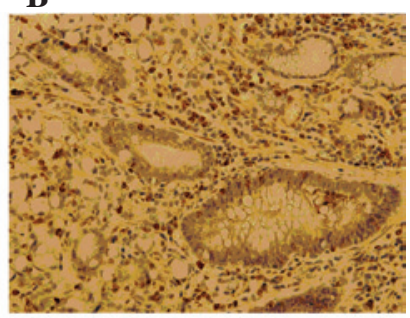

D

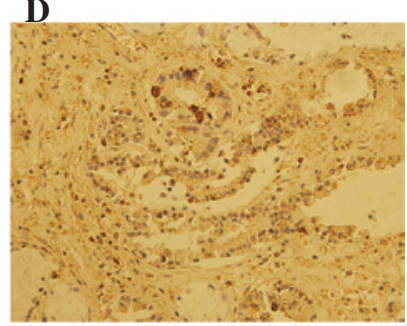

Figure 1. Expression of S100A4 in the gastric mucosa (magnification, x400). (A) Normal gastric mucosa. (B) Gastric mucinous adenocarcinoma. (C) Poorly differentiated gastric adenocarcinoma. (D) Villous gastric adenocarcinoma.

A

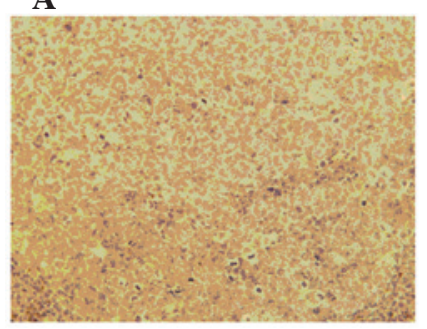

B

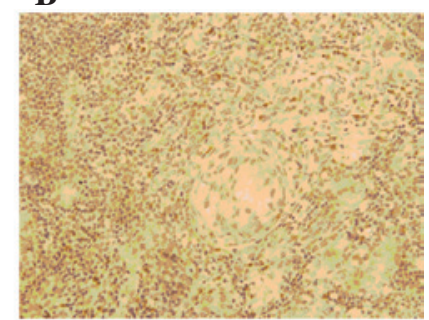

Figure 2. Expression of lymph node (magnification, $x 400$ ). (A) Non-metastatic lymph node, S100A4 . (B) Metastatic lymph node, S100A4+.

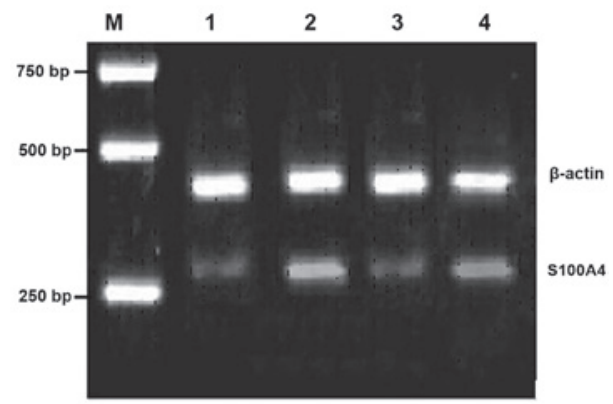

Figure 3. RT-PCR analysis of $\beta$-actin/S100A4 mRNA level in various tissues. M, DNA marker; 1, RT-PCR products from normal counterpart gastric tissues; 2, RT-PCR products of primary gastric cancer tissues; 3 , RT-PCR products from normal lymph node tissues; 4, RT-PCR products from positive lymph node tissues. RT-PCR, reverse transcription-polymerase chain reaction.

expression level of the metastatic LNs $(0.5491 \pm 0.0197)$ was higher than that in the normal LN tissues $(0.1558 \pm 0.0318)$. The difference between each group was statistically significant ( $\mathrm{P}<0.05$; Fig. 3).

Correlation between S100A4 expression and patient prognosis. In stage I-IV tumors, the 1- or 3-year survival rate of the patients with high S100A4 expression was significantly
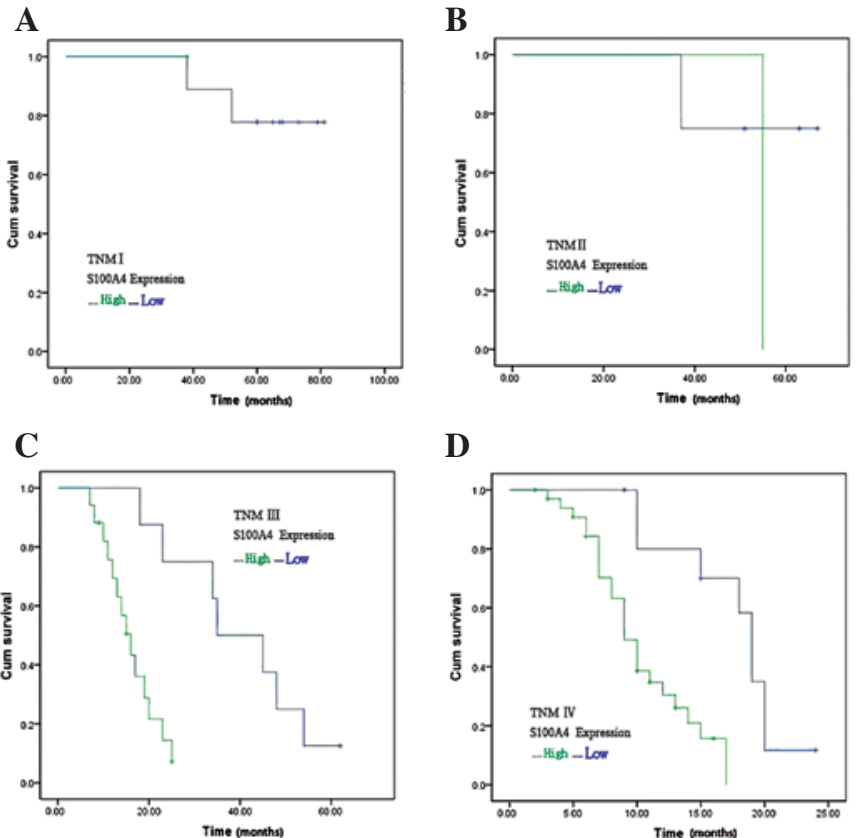

D

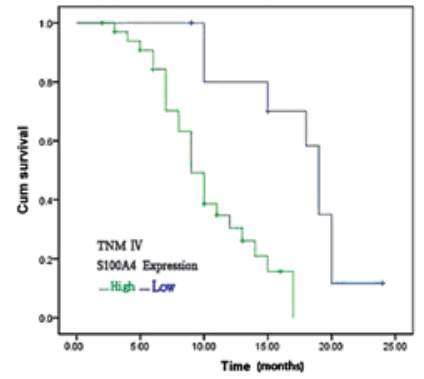

Figure 4. Kaplan-Meier curves with univariate analysis (log rank) for patients with low S100A4 expression vs. high S100A4 expression. (A) Stage I tumor. (B) Stage II tumor. (C) Stage III tumor. (D) Stage IV tumor. TNM, tumornode-metastasis.

lower than that in the patients with low S100A4 expression $(\mathrm{P}<0.05$; Fig. 4).

Multivariate analysis of clinicopathological parameters and prognosis. The factors with possible prognostic effects on young GC patients were analyzed by Cox regression. The results showed that patient age $(\mathrm{P}=0.035)$, tumor size $(\mathrm{P}=0.002)$, TNM stage $(\mathrm{P}=0.001)$ and $\mathrm{S} 100 \mathrm{~A} 4$ upregulation $(\mathrm{P}=0.000)$ were independent prognostic indicators for the disease (Table III).

\section{Discussion}

A number of studies on the clinicopathological and molecular biological features of GC in the elderly have been performed. However, only a few studies with a small sample size have analyzed GC in young patients (11). Previous studies have associated S100A4 protein expression with survival in several tumor types, including bladder (6), colorectal (7), ovarian (8) and esophageal carcinoma (9). S100A4 protein expression has also been revealed to have prognostic significance in GC (12-14). In a study of 436 cases, Wang et al (12) demonstrated that IHC staining for S100A4 is associated with LN metastasis and poor prognosis in GC patients. The present study demonstrates the prognostic significance of S100A4 overexpression in the tumor cells of young GC patients for the first time. Multivariate analysis showed that S100A4 overexpression was associated with patient outcome. The observed results may translate into clinically important differences.

In certain studies $(11,15)$, a female predominance was observed among young GC patients. However, a higher proportion of male patients has been noted in elderly GC patients (16). 
Table II. Association between S100A4 expression and LN metastasis.

\begin{tabular}{|c|c|c|c|c|}
\hline \multirow[b]{2}{*}{ Characteristics } & \multicolumn{2}{|c|}{ S100A4 } & \multirow[b]{2}{*}{$\chi^{2}$} & \multirow[b]{2}{*}{ P-value } \\
\hline & Low & High & & \\
\hline LN metastasis & & & 14.409 & 0.000 \\
\hline Negative & 45 & 17 & & \\
\hline Positive & 24 & 38 & & \\
\hline No. of LN metastases & & & 14.409 & 0.000 \\
\hline $1-6$ & 13 & 2 & & \\
\hline $7-15$ & 7 & 15 & & \\
\hline$>15$ & 4 & 21 & & \\
\hline
\end{tabular}

LN, lymph node.

Table III. Multivariate Cox regression survival analysis of clinicopathological parameters and S100A4.

\begin{tabular}{|c|c|c|c|c|c|}
\hline Parameters & B & SE & Wald & $\operatorname{Exp}(B)$ & P-value \\
\hline Age & 0.072 & 0.034 & 4.467 & 0.930 & 0.035 \\
\hline Gender & 0.350 & 0.361 & 0.937 & 1.419 & 0.333 \\
\hline Location & 0.204 & 0.246 & 0.684 & 0.816 & 0.408 \\
\hline Size & 1.474 & 0.471 & 9.799 & 4.369 & 0.002 \\
\hline Operation & 0.221 & 0.245 & 0.816 & 1.248 & 0.366 \\
\hline Lauren & 0.417 & 0.440 & 0.897 & 1.517 & 0.344 \\
\hline Histology & 0.069 & 0.178 & 0.151 & 0.933 & 0.698 \\
\hline Differentiaiton & 0.560 & 0.413 & 1.834 & 1.750 & 0.176 \\
\hline Borrmann & 0.116 & 0.377 & 0.095 & 0.890 & 0.758 \\
\hline TNM & 1.510 & 0.441 & 11.711 & 4.525 & 0.001 \\
\hline Metastasis & 1.391 & 0.896 & 2.414 & 4.021 & 0.120 \\
\hline H. pylori & 0.315 & 0.332 & 0.899 & 1.370 & 0.343 \\
\hline Expression & 2.315 & 0.507 & 20.864 & 10.129 & 0.000 \\
\hline
\end{tabular}

TNM, tumor-node-metastasis.

In the present study, the female-to-male ratio was 1.5:1 in the young GC patients. The reason for the higher number of female patients is not yet known. In the present study, no significant difference in S100A4 expression was observed between the male and female patients $(\mathrm{P}>0.05)$.

Infection caused by $H$. pylori is considered to be an important epidemiological risk factor for GC patients of all ages $(17,18)$. Moreover, certain epidemiological data have revealed an association between $H$. pylori infection and an increased risk of GC presenting at a young age $(19,20)$. However, in the present study, no significant difference in S100A4 expression was observed in the patients with and without $H$. pylori infection. In addition, $H$. pylori positivity was infrequently observed among the young GC patients.

Chung et al (15) reported that the incidence of primary lesions in the upper third of the stomach is higher in young patients than in elderly patients. In the present study, the same results were reported, although no significant difference was observed.
Similar to the results of previous studies $(11,15)$, the histology in young patients was observed to be more poorly differentiated.

Gastrectomy in combination with lymphadenectomy is the only potentially curative treatment for localized gastric carcinomas, and curative resection offers the only chance of long-term survival. However, radical resection is not common in young GC patients due to clinicopathological characteristics. Numerous studies have reported that the curative resection rate in young patients with GC is low. In the present study, the type of surgery showed no significant effect on S100A4 expression.

In the present study, quantitative RT-PCR revealed the presence of S100A4 mRNA in non-tumor mucosal samples from young patients. The non-metastatic LNs expressed S100A4 mRNA at low levels. The levels of S100A4 mRNA in the GC tissues and metastatic LNs were significantly higher compared with those in the matched normal gastric mucosa and non-metastatic LNs, respectively. Similarly, S100A4 expression was also detected using IHC. IHC showed that 
the S100A4 protein was associated with GC cells and other non-parenchymal cell types including lymphocytes. The proportion of specimens with epithelial cells stained for S100A4 increased between the GC tissues and metastatic LNs. However, no significant difference was observed. By contrast, the proportion of lymphocytes stained for S100A4 remained at a consistently high level in the normal gastric tissues, GC tissues and metastatic LNs. Significant differences in the expression levels of the S100A4 protein were observed among the TNM stages of GC, as well as between metastatic GC and non-metastatic GC. The results of the present study are consistent with the results of previous studies (12-14), in which S100A4 expression detected via IHC was increased in malignant relative to non-malignant GC specimens. Moreover, the deeper and more invasive regions of the specimens in these studies were enhanced in immunohistochemically detectable S100A4. This suggests that high levels of S100A4 protein are associated with the invading regions of the tumors. S100A4 overexpression was more frequently observed in the metastatic LNs than in the primary GC tissues from young patients. However, no significant difference was observed.

Previous studies reported that S100A4 overexpression is closely correlated with several factors for GC aggressiveness, such as LN metastasis, distant metastasis and TNM stage (12-14). In the present study, similar results were observed in the young GC patients. These data further support a significant correlation between S100A4 overexpression and GC progression, indicating the putative role of S100A4 in tumor cell aggressiveness.

In conclusion, S100A4 overexpression is an independent predictor of adverse prognosis in young GC patients. Thus, it may be used as a biomarker in future clinical studies.

\section{Acknowledgements}

This work was supported by the China National Natural Science Fund Committee (Grant No. 30971421) and Tianjin Natural Science Fund Committee (Grant No. 10JCYBJC10200).

\section{References}

1. Kamangar F, Dores GM and Anderson WF: Patterns of cancer incidence, mortality, and prevalence across five continents: defining priorities to reduce cancer disparities in different geographic regions of the world. J Clin Oncol 24: 2137-2150, 2006 .
2. Simsa J, Leffler J, Hoch J, et al: Gastric cancer in young patients - is there any hope for them. Acta Chir Belg 104: 673-676, 2004.

3. Chen CY, Wu CW, Lo SS, et al: Peritoneal carcinomatosis and lymph node metastasis are prognostic indicators in patients with Borrmann type IV gastric carcinoma.Hepatogastroenterology 49: 874-877, 2002

4. Fareed KR, Kaye P, Soomro IN, et al: Biomarkers of response to therapy in oesophago-gastric cancer. Gut 58: 127-143, 2009.

5. Garrett SC, Varney KM, Weber DJ and Bresnick AR: S100A4, a mediator of metastasis. J Biol Chem 281: 677-680, 2006.

6. Matsumoto K, Irie A, Satoh T, et al: Expression of S100A2 and S100A4 predicts for disease progression and patient survival in bladder cancer. Urology 70: 602-607, 2007.

7. Sack U, Walther W, Scudiero D, et al: Novel effect of antihelminthic Niclosamide on S100A4-mediated metastatic progression in colon cancer. J Natl Cancer Inst 103: 1018-1036, 2011.

8. Kikuchi N, Horiuchi A, Osada R, et al: Nuclear expression of S100A4 is associated with aggressive behavior of epithelial ovarian carcinoma: an important autocrine/paracrine factor in tumor progression. Cancer Sci 97: 1061-1069, 2006.

9. Lee OJ,Hong SM, Razvi MH, et al: Expression of calcium-binding proteins S100A2 and S100A4 in Barrett's adenocarcinomas. Neoplasia 8: 843-850, 2006.

10. Flatmark K, Pedersen KB, Nesland JM, et al: Nuclear localization of the metastasis-related protein S100A4 correlates with tumour stage in colorectal cancer. J Pathol 200: 589-595, 2003.

11. Medina-Franco H, Heslin MJ and Cortes-Gonzale R: Clinicopathological characteristics of gastric carcinoma in young and elderly patients: a comparative study. Ann Surg Oncol 7: 515-519, 2000

12. Wang YY, Ye ZY, Zhao ZA, et al: High-level expression of S100A4 correlates with lymph node metastasis and poor prognosis in patients with gastric cancer. Ann Surg Oncol 17: 89-97, 2010.

13. Cho YG, Nam SW, Kim TY, et al: Overexpression of S100A4 is closely related to the aggressiveness of gastric cancer. APMIS 111: 539-545, 2003.

14. Yonemura Y, Endou Y, Kimura K, et al: Inverse expression of S100A4 and E-cadherin is associated with metastatic potential in gastric cancer. Clin Cancer Res 6: 4234-4242, 2000.

15. Chung HW, Noh SH and Lim JB: Analysis of demographic characteristics in 3242 young age gastric cancer patients in Korea. World J Gastroenterol 16: 256-263, 2010.

16. Kim DY, Joo JK, Ryu SY, et al: Clinicopathologic characteristics of gastric carcinoma in elderly patients: A comparison with young patients. World J Gastroenterol 11: 22-26, 2005.

17. Chung HW, Park SW, Chung JB, et al: Differences in genetic expression profiles between young-age and old-age gastric adenocarcinoma using cDNA microarray for endocrine disruptor study. Oncol Rep 12: 33-39, 2004.

18. Ershler WB and Longo DL: The biology of aging: the current research agenda. Cancer 80: 1284-1293, 1997.

19. Masuda G, Tokunaga A, Shirakawa T, et al: Helicobacter pylori infection, but not genetic polymorphism of CYP2E1, is highly prevalent in gastric cancer patients younger than 40 years. Gastric Cancer 10: 98-103, 2007.

20. Kokkola A, Valle J, Haapiainen R, et al: Helicobacter pylori infection in young patients with gastric carcinoma. Scand J Gastroenterol 31: 643-647, 1996. 